\title{
Endovascular Repair for Abdominal Aortic Aneurysm with an Ectopic Pelvic Kidney: Case Report and Procedural Consideration of the Aberrant Renal Artery
}

\author{
Kenji Sakai, Taiju Watanabe, Tetsuya Yoshida* \\ Department of Cardiovascular Surgery, Hokushin General Hospital, Nagano, Japan \\ Email: ^tyoshida-cvs@umin.ac.jp
}

How to cite this paper: Sakai, K., Watanabe, T. and Yoshida, T. (2018) Endovascular Repair for Abdominal Aortic Aneurysm with an Ectopic Pelvic Kidney: Case Report and Procedural Consideration of the Aberrant Renal Artery. World Journal of Cardiovascular Surgery, 8, 111-116.

https://doi.org/10.4236/wjcs.2018.86010

Received: May 6, 2018

Accepted: June 15, 2018

Published: June 19, 2018

Copyright $\odot 2018$ by authors and Scientific Research Publishing Inc. This work is licensed under the Creative Commons Attribution International License (CC BY 4.0).

http://creativecommons.org/licenses/by/4.0/

\begin{abstract}
A 91-year-old female presented with a pulsatile abdominal mass. Her past medical history included hypertension and hyperlipidemia. A $6.9 \mathrm{~cm}$ infrarenal abdominal aortic aneurysm, with a left ectopic pelvic kidney and two aberrant renal arteries, one each from the left and right common iliac arteries was found on computed tomography. Because of the high risk of rupture, surgery was recommended and an endovascular aneurysm repair was performed. The antegrade flow of the aberrant renal artery from the left common iliac artery was preserved. The right aberrant renal artery was covered with stent graft. The patient's serum creatinine level remained unchanged throughout the postoperative course, with an uneventful postoperative recovery.
\end{abstract}

\section{Keywords}

Aberrant Renal Artery, Ectopic Pelvic Kidney, Endovascular Abdominal Aortic Aneurysm Repair

\section{Introduction}

An ectopic pelvic kidney is a congenital abnormality, in which the kidney fails to ascend at embryologic development and remains in pelvis [1]. The incidence of ectopic pelvic kidney was reported to be between $0.033 \%$ and $0.047 \%$ [2], and the occurrence in combination with abdominal aortic aneurysm (AAA) should be extremely rare. There are a limited number of reports in literature about AAA with an ectopic pelvic kidney. One or two aberrant renal arteries are often ob- 
served, originating from various parts of artery [3]. The ectopic pelvic kidney is often asymptomatic [4], but the anatomical characteristics make it difficult to perform surgery. We report a case of an AAA with ectopic pelvic kidney, treated by endovascular abdominal aortic aneurysm repair (EVAR), along with a review of the literature.

The patient described in this case report consented to submission of her case.

\section{Case Report}

A 91-year-old Japanese woman was referred to our outpatient department with a pulsatile abdominal mass. Physical examination showed systolic hypertension (220/116 mmHg). Her medical history included hyperlipidemia, arterial hypertension, chronic kidney disease (CKD), and reflux esophagitis, but no history of any allergies. Her family history was noncontributory.

A computed tomography (CT) scan showed an infrarenal AAA with a diameter of $69 \mathrm{~mm}$, a left ectopic pelvic kidney, and two aberrant renal arteries, one each from the left and right common iliac arteries (CIA) (Figure 1). The diameter of right aberrant renal artery was $1.5 \mathrm{~mm}$, and that of the left was $2.5 \mathrm{~mm}$.

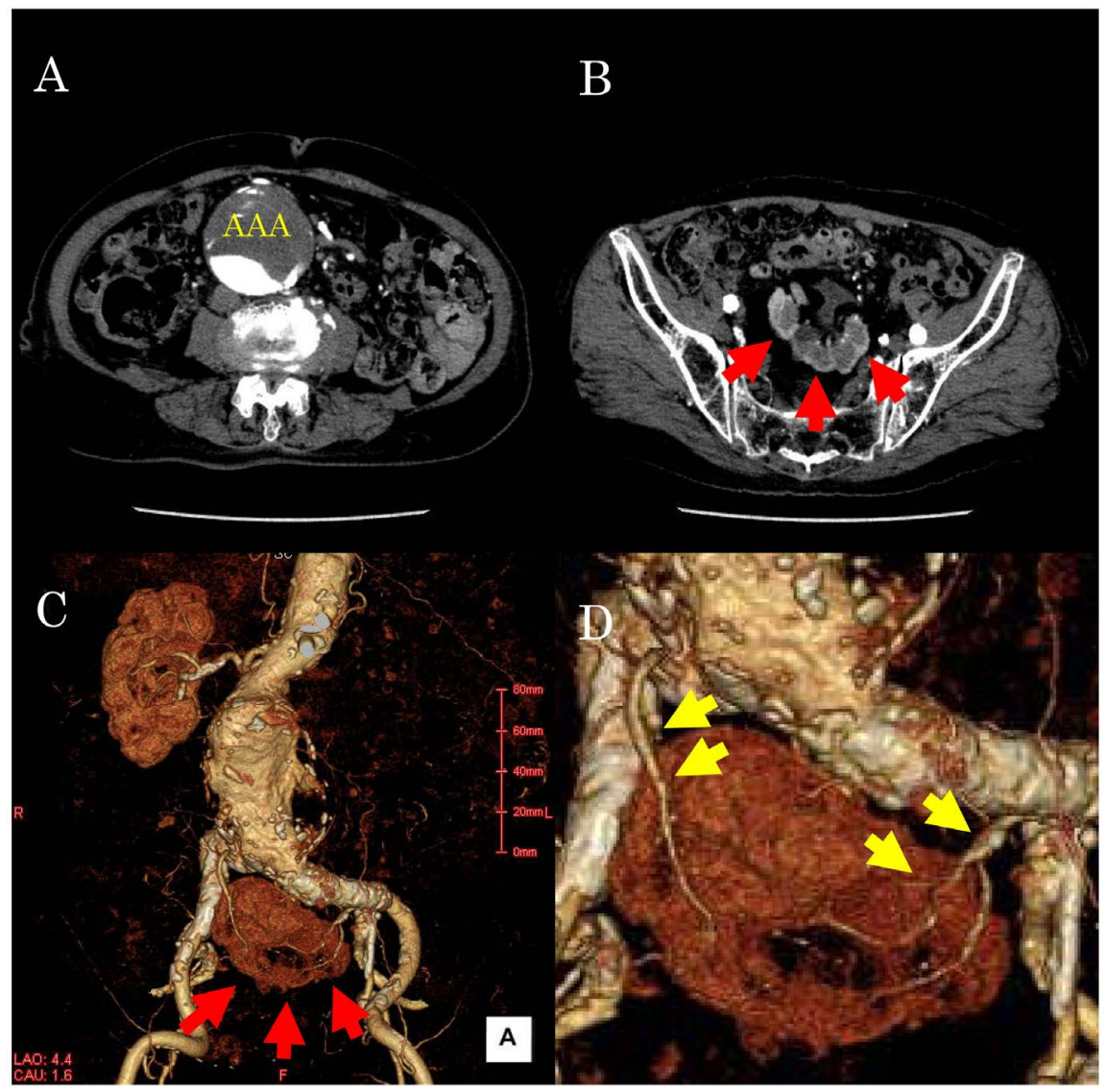

Figure 1. Computed tomography (CT) scan showed infrarenal abdominal aortic aneurysm with a diameter of $69 \mathrm{~mm}$ (A), and ectopic pelvic kidney (B: red arrow). 3D-CT showed left ectopic pelvic kidney and two aberrant renal arteries $(C)$. One aberrant artery each, from the left and right common iliac arteries (D: yellow arrow). 
Laboratory investigation showed elevated serum creatinine level $(1.51 \mathrm{mg} / \mathrm{dL})$. The estimated glomerular filtration rate (eGFR) was $25 \mathrm{ml} / \mathrm{min} / 1.73 \mathrm{~m}^{2}$.

The diagnosis was AAA with ectopic pelvic kidney.

Although she was 91-year-old, her activities of daily living were independent. Due to significant arterial hypertension, the risk of aneurysmal rupture was high, and surgical treatment was recommended.

The anatomical conditions of abdominal aorta and iliac arteries were within instruction for use of all commercially available EVAR devices.

Preservation of the aberrant artery and renal protection was necessary due to the history of CKD. The aberrant renal artery from the right CIA originated near the bifurcation of the aorta; hence, it was difficult to preserve the antegrade blood flow after EVAR. The aberrant renal artery from left CIA originated from the distal part of CIA. Our strategy was that the left aberrant renal artery was preserved, and the right aberrant renal artery covered with stent graft. The Gore Excluder stent-grafts (W.L. Gore \& Associates, Newark, DE, USA) were successfully implanted.

Postoperatively, she recovered well without complications, and was discharged from the hospital nineteen days after EVAR.

We performed a follow-up CT at 3 months after EVAR. The aberrant renal artery from left CIA and ectopic pelvic kidney revealed enhanced contrast (Figure 2). The aberrant renal artery from right CIA was enhanced in the retrograde direction, and there was no type 2 endoleak from the artery. One year later, laboratory investigation revealed that the creatinine level was stable. At the time of her most recent follow-up, the patient reported feeling well without any complaints.

\section{Discussion}

The kidney originates in the pelvis early in fetal life, and gradually ascends to

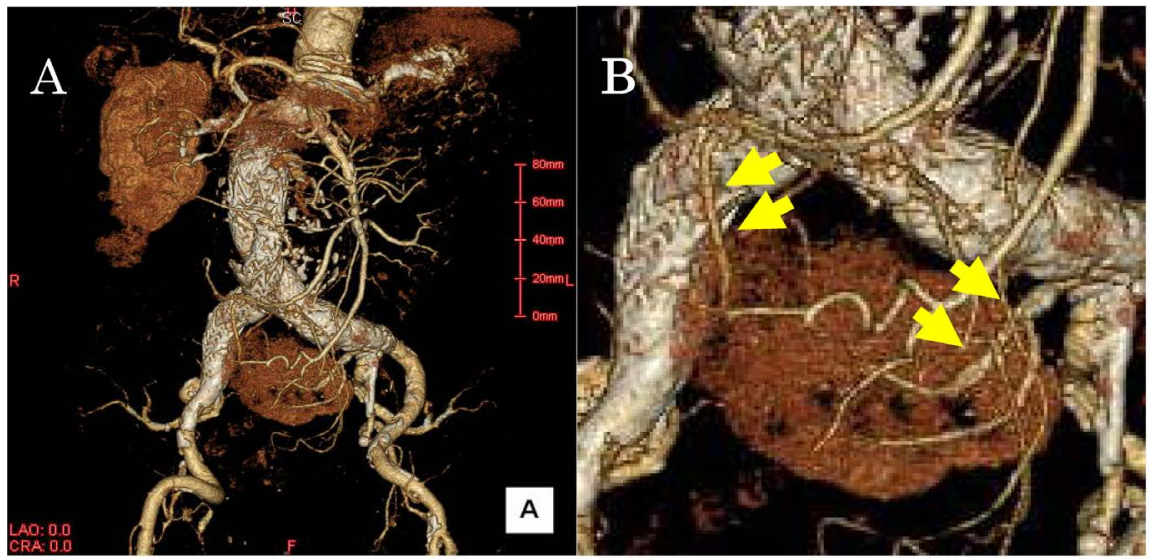

Figure 2. Postoperative 3D-Computed tomography scan showed patency of the aberrant renal artery from left common iliac artery (CIA) and good enhancement of ectopic pelvic kidney (A) and (B). The aberrant renal artery from right CIA was enhanced in the retrograde direction, and there was no type 2 endoleak from the right aberrant renal artery (B: yellow arrow). 
reach the normal position. While ascending, each kidney acquires blood supply from the neighboring vessels, initially from the external iliac artery (EIA) and internal iliac artery (IIA), and directly from the aorta at the eighth week of embryologic development [1].

Ectopic pelvic kidney is a condition that results when the kidneys fail to ascend to their normal position during early embryologic development. A simple ectopic kidney is usually asymptomatic and may function normally [4].

There is some variation in the blood supply of an ectopic pelvic kidney. The aberrant artery can originate from the abdominal aorta, CIA or EIA [3]. There can be various combinations of the aberrant artery. Before initiating the surgical procedure for AAA, evaluation of the aberrant artery is important.

In this case, the left ectopic pelvic kidney was accidentally detected on CT scan. Two aberrant renal arteries were observed, one each from the left and right CIA. The diameter of the right aberrant renal artery was thinner than that of the left artery.

Revascularization of aberrant artery and renal protection becomes a challenge during the surgery for AAA with an ectopic pelvic kidney. Since renal artery is an anatomically terminal artery, there is a risk of renal infarction due to blood flow obstruction.

Hemodynamics of the two aberrant renal arteries are similar to those of the accessory renal artery. There is a consensus about the treatment of accessory renal artery during AAA surgery; however, there is no consensus about the treatment of aberrant renal artery when operating for AAA. The Society of Vascular Surgery Consensus statement for the treatment of AAA recommends preservation and reimplantation of a major accessory renal artery $(>3 \mathrm{~mm})$ or those that supply one-third or more of the kidney, at the time of open aneurysmal repair [5].

Greenberg JI et al. reported the long-term results after coverage of the accessory artery during EVAR. They reported that coverage of an accessory renal artery larger than $3 \mathrm{~mm}$ may cause local renal infarction, but renal function is maintained and there was no difference in 30 day or 24 months survival [6]. Reconstruction of the accessory renal artery during EVAR is controversial. Before deciding on the surgical approach, a preoperative detailed study of the CT scan images is necessary. This can help in selection of the best surgical method and avoid intraoperative and postoperative complications [7].

Recently, EVAR with fenestrated device and hybrid surgery were adapted for AAA complicated with ectopic kidney, and good clinical outcomes were reported [8] [9] [10] [11].

The aberrant renal artery originated from the aneurysmal sac or from the aortic bifurcation in the previous report. In these cases, an additional maneuver is necessary to salvage at least one aberrant renal artery.

In our case, two aberrant renal arteries were observed, and the sizes were 1.5 $\mathrm{mm}$ and $2.5 \mathrm{~mm}$ respectively. Each aberrant renal artery supplied about $50 \%$ of 
the kidney. Right aberrant artery could have been covered during EVAR. However, since the left aberrant artery originated from the distal part of left CIA, it was possible to preserve the antegrade blood flow during simple EVAR.

We did not use the fenestrated device, and preserved the thicker aberrant renal artery. The thinner aberrant artery was covered with stent graft. The post-operative renal function was unchanged, and good clinical outcome was achieved. As far as we searched the literature, this is the first report for coverage of one aberrant renal artery during EVAR, to treat of AAA with ectopic pelvic kidney.

There are, however, only a few reports about EVAR in the treatment of AAA with ectopic pelvic kidney. More cases are necessary to validate the optimal method of treatment. A longer observational period and more case studies are warranted.

\section{Conclusion}

AAA complicated with ectopic pelvic kidney is rare. Preoperative evaluation with CT scan was helpful in planning the surgical procedure. We performed EVAR and sacrificed the thinner aberrant renal artery. In this case, the postoperative renal function was not affected, and a good clinical outcome was achieved.

\section{References}

[1] Kumar, S., Bolla, S.R. and Vollala, V.R. (2011) Unilateral Ectopic Kidney in the Pelvis-A Case Report. Chang Gung Medical Journal, 34, 10-12.

[2] Murakami, T., Makino, Y., Suto, Y. and Yasuda, K. (2004) Abdominal Aortic Aneurysm Repair in a Patient with a Congenital Solitary Pelvic Kidney. A Case Report. The Journal of Cardiovascular Surgery, 45, 501-504.

[3] Glock, Y., Blasevich, R., Laghzaoui, A., Roux, D. and Fournial, G. (1997) Abdominal Aortic Aneurysm and Congenital Pelvic Kidney. A Rare Association. The Texas Heart Institute, 24, 131-133.

[4] Bhoil, R., Sood, D., Singh, Y.P., Nimkar, K. and Shukla, A. (2015) An Ectopic Pelvic Kidney. Polish Journal of Radiology, 80, 425-427. https://doi.org/10.12659/PJR.894603

[5] Chaikof, E.L., Brewster, D.C., Dalman, R.L., Makaroun, M.S., Illig, K.A., Sicaard, G.A., et al. (2009) The Care of Patients with an Abdominal Aortic Aneurysm: The Society for Vascular Surgery Practice Guidelines. Journal of Vascular Surgery, 50, S2-49. https://doi.org/10.1016/j.jvs.2009.07.002

[6] Greenberg, J.I., Dorsey, C., Dalman, R.L., Lee, J.T., Harris, E.J., Hernandez-Boussard, T., et al. (2012) Long-Term Results after Accessory Renal Artery Coverage during Endovascular Aortic Aneurysm Repair. Journal of Vascular Surgery, 56, 291-296. https://doi.org/10.1016/j.jvs.2012.01.049

[7] De Caridi, G., Massara, M., Greco, M., Mastrojeni, C., Serra, R., Salomone, I., et al. (2015) Surgical Treatment of a Voluminous Infrarenal Abdominal Aortic Aneurysm with Horseshoe Kidney: Tips and Tricks. Annals of Vascular Diseases, 8, 324-327. https://doi.org/10.3400/avd.cr.15-00083 
[8] Mastracci, T.M., Greenberg, R.K. and Pfaff, K. (2008) EVAR of a Large AAA with a Pelvic Kidney. Endovascular Today, 40-42.

[9] Morales, J.P. and Greenberg, R.K. (2009) Customised Stent Graft for Complex Thoraco-Abdominal Aneurysm Associated with Congenital Pelvic Kidney. European Journal of Vascular and Endovascular Surgery, 37, 557-559. https://doi.org/10.1016/j.ejvs.2009.01.006

[10] Machado, R., Silveira, D., Almeida, P. and Almeida, R. (2015) Hybrid Surgery as a New Perspective for Treatment of Abdominal Aortic Aneurysm Associated with a Congenital Pelvic Kidney. Angiologia e Cirurgia Vascular, 11, 11-14. https://doi.org/10.1016/j.ancv.2014.12.004

[11] Malinowski, M.J., Al-Nouri, O., Hershberger, R., Halandras, P.M., Aulivola, B. and Cho, J.S. (2014) Abdominal Aortic Aneurysm Associated with Congenital Solitary Pelvic Kidney Treated with Novel Hybrid Technique. Annals of Vascular Surgery, 28, 1566.e7-e10. https://doi.org/10.1016/j.avsg.2014.01.015 